\title{
Afamin: an early predictor of preeclampsia
}

\author{
Angela Köninger ${ }^{1} \cdot$ Antje Enekwe $^{1} \cdot$ Pawel Mach $^{1} \cdot$ Dimitrios Andrikos ${ }^{1} \cdot$ Boerge Schmidt $^{2} \cdot$ Mirjam Frank $^{2}$. \\ Cahit Birdir $^{1,3} \cdot$ Rainer Kimmig $^{1} \cdot$ Alexandra Gellhaus $^{1} \cdot$ Hans Dieplinger $^{4}(\mathbb{D}$
}

Received: 14 May 2018 / Accepted: 6 September 2018 / Published online: 15 September 2018

(c) The Author(s) 2018

\begin{abstract}
Purpose Oxidative stress is involved in the pathogenesis of hypertensive disorders such as preeclampsia (PE) and associated with the human vitamin E-binding protein afamin. The aim of this study was, therefore, to analyse afamin in the first trimester of patients developing PE later in pregnancy and in control subjects without pregnancy complications.

Methods In this retrospective study, 137 serum samples from the first trimester of pregnancy were analysed in a case-control study design. 39 patients developed PE (10 patients with early-onset and 29 patients with late onset disease) and 98 women had an uncomplicated pregnancy. Mann-Whitney $U$ test, $t$ test, logistic regression and ROC analyses were performed for statistical evaluation.

Results Pregnant women developing PE presented with higher afamin concentrations in the first trimester [median $101.81 \mathrm{mg} / \mathrm{L}$; interquartile range (IQR) 88.94-113.26] compared to subjects with uncomplicated pregnancy (median 86.40; IQR 75.26-96.92; $p<0.001$ ). After adjusting for confounders, the odds ratio per afamin standard deviation was 1.60 (95\% CI: $1.04-2.58 ; p=0.04)$. An afamin threshold concentration of $87.8 \mathrm{mg} / \mathrm{L}$ exhibited the best sensitivity $(79.5 \%)$ and specificity $(57.1 \%)$ in predicting PE. Subgroup analysis of early- and late-onset disease resulted in substantially higher afamin concentrations in women with developing late-onset PE compared to controls $(p<0.001)$ with an odds ratio per afamin standard deviation of 1.62 (95\% CI: $0.98-2.70 ; p=0.06$ ).

Conclusions Serum afamin concentrations are elevated in the first trimester among patients developing PE compared to controls. Substantial differences were observed mainly among patients with late-onset PE.
\end{abstract}

Keywords Afamin $\cdot$ Preeclampsia $\cdot$ Early and late onset $\cdot$ Prediction

Hans Dieplinger

hans.dieplinger@i-med.ac.at

1 Department of Gynaecology and Obstetrics, University of Duisburg-Essen, Hufelandstrasse 55, 45122 Essen, Germany

2 Institute for Medical Informatics, Biometry and Epidemiology (IMIBE), University of Duisburg-Essen, Hufelandstraße 55, 45122 Essen, Germany

3 Department of Gynaecology and Obstetrics, University Hospital Carl Gustav Carus, Fletscherstraße 74, 01307 Dresden, Germany

4 Division of Genetic Epidemiology, Department of Medical Genetics, Molecular and Clinical Pharmacology, Medical University of Innsbruck, Schöpfstraße 41, 6020 Innsbruck, Austria

\section{Introduction}

Preeclampsia (PE) and other hypertensive disorders during pregnancy occur in $\sim 2-8 \%$ of pregnant women [1]. Currently, no treatment exists to prevent the progression of the disease after its clinical diagnosis. However, the occurrence of placental disorders can be prevented by the administration of Aspirin ${ }^{\circledR}$ before the 16th week of pregnancy in women with clinical risk factors for PE such as pathological Doppler indices, positive family history or with $\mathrm{PE}$ in a previous pregnancy [2] as well as in primigravidae screened to be at high risk [3]. Elevated risk can be determined in the first trimester by screening for the biochemical markers, pregnancyassociated plasma protein A (PAPP-A) and placental growth factor (PIGF), as well as for biophysical markers such as maternal mean arterial blood pressure and pulsatility indices of the uterine arteries $[4,5]$. 
$\mathrm{PE}$ is characterised as an early-onset form, which occurs before the 34th week of pregnancy, and a late-onset form, which occurs after 34 weeks of pregnancy. The prediction [4] as well as prevention [2, 3] of early-onset PE is much more successful compared to the late-onset form suggesting different pathophysiological mechanisms [6].

While early-onset PE is thought to result from disturbed trophoblast invasion, the late-onset form generally seems to result from adverse maternal conditions [7]. In summary, reliable tools have been established to screen and prevent early-onset PE, whereas the late-onset form still needs further research regarding prediction and prevention.

For this reason, our research focused on maternal conditions which may contribute to the pathophysiology leading to placental dysfunction and PE, mainly in the late-onset form. It is already well known that PE is strongly associated with a maternal state of endothelial dysfunction, chronic inflammation, and oxidative stress [8-11]. Women suffering from placental dysfunction subsequently develop metabolic syndrome and insulin resistance (IR) at a frequency of 20 and $60 \%$, respectively [8].

In the present work, we have investigated the role of afamin in the development of PE. Afamin has been previously described as a vitamin E-binding glycoprotein from human plasma which is also found in extravascular fluids [12]. It is a member of the albumin gene family [13]. Vita$\min \mathrm{E}$ is an antioxidant nutrient that prevents the oxidation of lipids [14]. In contrast to human plasma, a substantial association between afamin concentrations and vitamin $\mathrm{E}$ concentrations has been detected in extravascular fluids like cerebrospinal and follicular fluids [15]. It has been previously reported that serum afamin concentrations are increased in response to various conditions of oxidative stress: afamin concentrations are elevated in the peritoneal fluid of women with endometriosis [16], and are strongly associated with the development of metabolic syndrome including a strong correlation with body mass index (BMI) [17]. A recently published population-based study including more than 20,000 individuals demonstrated elevated afamin concentrations in individuals with type 2 diabetes mellitus (T2DM) and IR [18]. Serum afamin concentrations are elevated in patients with polycystic ovarian syndrome (PCOS) and IR $[19,20]$ and in the first trimester of women developing subsequent gestational diabetes mellitus $[21,22]$. These observations agree with the results of several studies that have suggested cross-links between oxidative stress, the insulin pathway, chronic inflammation and endothelial dysfunction in the pathogenesis of PE [23-26]. Afamin concentrations are known to rise continuously with increasing gestational age [27]. Since afamin is not synthesised by the placenta [27], its concentrations reflect most likely a maternal condition rather than a placental disease.
The aim of this study was to evaluate a possible association between PE, especially in the late-onset form, and concentrations of afamin as an indicator of oxidative stress by comparing afamin concentrations during the first trimester in the serum of pregnant women subsequently developing PE with matched healthy controls without pregnancy complications.

\section{Materials and methods}

\section{Study design and subjects}

Patients and control subjects were analysed retrospectively in a nested case-control study design (pre-eclamptic patients: $n=39$; controls: $n=98$ ) recruited from a consecutively performed first-trimester screening programme at the Department of Obstetrics and Gynaecology of the University Hospital of Essen for obstetric care between 2002 and 2012. Patients and healthy controls were selected from available stored blood samples.

Blood samples were collected between week $11+0$ and week $13+6$ of pregnancy. All patients had singleton pregnancies. Patients with gestational diabetes, pre-existing diabetes, chromosomal anomalies or preterm delivery except because of PE were excluded from the study. PE was defined according to the guidelines of the American College of Obstetricians and Gynecologists for hypertension in pregnancy, i.e. proteinuria of $\geq 300 \mathrm{mg} / 24 \mathrm{~h}$ and elevated blood pressure with diastolic values $\geq 90 \mathrm{mmHg}$ and systolic values $\geq 140 \mathrm{mg}$ (measured at least twice within 4-6 h) after the 20th week of pregnancy [28]. Controls were selected from pregnant subjects who did not develop PE or related outcomes during pregnancy.

From the 39 patients who developed PE during the course of pregnancy, 10 patients had early-onset PE and 29 patients had late-onset PE. Patient characteristics are shown in Table 1.

\section{Sample collection and laboratory parameters}

$9 \mathrm{ml}$ of blood was taken from every patient with the S-Monovette Blood Collection System (Sarstedt AG and Co., Nürnbrecht, Germany) for subsequent determination of parameters of interest. Blood samples were immediately stored at $4{ }^{\circ} \mathrm{C}$ and processed within $4 \mathrm{~h}$ to avoid cell lysis. Blood fractionation was carried out by centrifugation at $2500 \times \mathrm{g}$ for $10 \mathrm{~min}$, and 3-4 $\mathrm{ml}$ of the supernatant constituting blood serum was removed and stored at $-80^{\circ} \mathrm{C}$. For afamin analysis, the samples were thawed, divided into aliquots and restored at $-80^{\circ} \mathrm{C}$. Frozen aliquots were sent to the Division of Genetic Epidemiology at Medical University of Innsbruck in 2016 for analysing afamin. Afamin concentrations were 
Table 1 Characteristics of patients with preeclampsia (PE) and patients with uncomplicated pregnancies (determined during the first trimester)

\begin{tabular}{|c|c|c|c|c|c|c|}
\hline $\begin{array}{l}\text { Mean (STD) } \\
\text { Median (IQR) }\end{array}$ & $\begin{array}{l}\text { Control subjects } \\
(N=98)\end{array}$ & $\operatorname{PE}(N=39)$ & $p$ value & Early-onset PE $(N=10)$ & Late-onset PE $(N=29)$ & $p$ value \\
\hline Age (years) & $\begin{array}{l}32.79(4.81) \\
33.00(29.00-37.00)\end{array}$ & $\begin{array}{l}33.15(4.97) \\
34.00(31.00-37.00)\end{array}$ & 0.69 & $\begin{array}{l}30.50(5.58) \\
31.00(30.00-33.00)\end{array}$ & $\begin{array}{l}34.07(4.48) \\
35.00(31.00-37.00)\end{array}$ & 0.05 \\
\hline $\operatorname{Afamin}(\mathrm{mg} / \mathrm{L})$ & $\begin{array}{l}86.90(17.06) \\
86.40(75.26-96.92)\end{array}$ & $\begin{array}{l}103.13(23.58) \\
101.81(88.94-113.26)\end{array}$ & $<0.001$ & $\begin{array}{l}95.56(16.45) \\
93.68(88.03-102.00)\end{array}$ & $\begin{array}{l}105.75(25.31) \\
105.10(89.78-116.17)\end{array}$ & 0.20 \\
\hline PAPP-A (ng/mL) & $\begin{array}{l}1.29(0.72) \\
1.13(0.74-1.76)\end{array}$ & $\begin{array}{l}1.70(2.05) \\
1.13(0.65-1.79)\end{array}$ & 0.84 & $\begin{array}{l}0.88(0.59) \\
0.80(0.62-1.11)\end{array}$ & $\begin{array}{l}1.99(2.30) \\
1.28(0.68-2.33)\end{array}$ & 0.06 \\
\hline PlGF (pg/mL) & $\begin{array}{l}41.97(18.59) \\
37.65(29.53-48.39)\end{array}$ & $\begin{array}{l}30.75(18.40) \\
27.08(19.07-36.05)\end{array}$ & $<0.001$ & $\begin{array}{l}29.86(22.61) \\
24.43(18.76-36.27)\end{array}$ & $\begin{array}{l}31.05(17.17) \\
28.38(20.15-35.90)\end{array}$ & 0.71 \\
\hline $\operatorname{BMI}\left(\mathrm{kg} / \mathrm{m}^{2}\right)$ & $\begin{array}{l}23.91(4.95) \\
22.90(20.90-25.00)\end{array}$ & $\begin{array}{l}30.45(7.40) \\
29.00(23.10-36.78)\end{array}$ & $<0.001$ & $\begin{array}{l}29.58(7.53) \\
28.85(23.10-35.00)\end{array}$ & $\begin{array}{l}30.75(7.47) \\
29.00(23.08-37.53)\end{array}$ & 0.85 \\
\hline $\begin{array}{l}\text { Gestational age at } \\
\text { blood sampling }(\mathrm{d})\end{array}$ & $\begin{array}{l}87(4) \\
87(85-90)\end{array}$ & $\begin{array}{l}88(5) \\
88(85-91)\end{array}$ & 0.19 & $\begin{array}{l}89(6) \\
87(85-95)\end{array}$ & $\begin{array}{l}88(4) \\
88(86-91)\end{array}$ & 0.53 \\
\hline $\begin{array}{l}\text { Gestational age } \\
\text { at delivery }(\mathrm{d}, \\
\text { weeks }+\mathrm{d})\end{array}$ & $\begin{array}{l}274(8) \\
39+1(1+1) \\
274(268-281) \\
39+1(38+2-40+1)\end{array}$ & $\begin{array}{l}249(28) \\
35+4(4+0) \\
256(245-266) \\
36+4(35+0-38+0)\end{array}$ & $<0.001$ & $\begin{array}{l}214(34) \\
30+4(4+6) \\
214(181-236) \\
30+4(25+6-33+5)\end{array}$ & $\begin{array}{l}261(11) \\
37+2(1+4) \\
257(253-268) \\
36+5(36+1-38+2)\end{array}$ & $<0.001$ \\
\hline Birth weight (g) & $\begin{array}{l}3417(425) \\
3378(3150-3640)\end{array}$ & $\begin{array}{l}2604(938) \\
2860(2286-3258)\end{array}$ & $<0.001$ & $\begin{array}{l}1388(966) \\
1345(450-2030)\end{array}$ & $\begin{array}{l}3024(430) \\
2985(2714-3429)\end{array}$ & $<0.001$ \\
\hline $\begin{array}{l}\text { Birth weight (percen- } \\
\text { tiles) [41] }\end{array}$ & $\begin{array}{l}50.21(25.27) \\
51.50(31.00-69.00)\end{array}$ & $\begin{array}{l}42.46(28.31) \\
42.00(18.50-61.00)\end{array}$ & 0.13 & $\begin{array}{l}24.90(32.58) \\
12.50(1.00-36.00)\end{array}$ & $\begin{array}{l}48.52(24.46) \\
51.00(26.75-64.25)\end{array}$ & 0.02 \\
\hline
\end{tabular}

Values are presented as means with standard deviations (STD); medians with interquartile ranges (IQR)

$B M I$ body mass index, $P A P P-A$ pregnancy-associated plasma protein A, $P l G F$ placental growth factor, $d$ days

measured with a double-antibody sandwich enzyme-linked immunosorbent assay (ELISA, BioVendor, Brno, Czech Republic) using two different monoclonal antibodies against human afamin as modified from a previously described protocol [29]. Recombinantly expressed and purified human afamin served as assay standard. According to the manufacturer's manual, within-run and run-to-run coefficients of variation were 3.6 and $3.4 \%$, respectively, at a mean afamin concentration of $80 \mathrm{mg} / \mathrm{L}$.

Concentrations of PAPP-A and PlGF were measured with automated fluorescence assays (BRAHMS PAPP-A, 866.075, PlGF plus, 859.075) on a BRAHMS KRYPTOR immune analyzer (BRAHMS Kryptor Compact, Thermo Fischer Scientific, BRAHMS GmbH, Henningsdorf, Germany) in the Department for Gynaecology and Obstetrics at the University of Essen, Germany.

\section{Statistical analyses}

Mann-Whitney $U$ test or $t$ test was performed to compare the parameters of interest between subgroups. Logistic regression was used to detect associations between afamin concentrations in the first trimester and subsequent PE. BMI and gestational age at blood sampling were included in the analysis as potential confounders, since afamin concentrations increase with gestational age [27] and BMI [17] as does the risk for PE. Afamin seems not to be synthesized by the placenta [27] and there is no evidence for a direct influence on afamin secretion through placental-derived substances. Thus, BMI and gestational age, but not PIGF and PAPP-A, were considered as confounders in the analysis.

Receiver operating characteristic (ROC) analysis was used to test the ability of serum afamin concentrations to discriminate between pregnant women developing PE and those not developing PE. All statistical analyses were performed with the R statistical package, version 3.4.0 [30].

\section{Results}

\section{Serum afamin concentrations determined during the first trimester}

Patient characteristics are shown in Table 1. Patients suffering from $\mathrm{PE}$ and women with uncomplicated pregnancies differed significantly regarding BMI, PIGF, gestational age at delivery and newborn weight.

Median serum afamin concentrations during the first trimester in patients developing PE were significantly higher (median $101.81 \mathrm{mg} / \mathrm{L}$; interquartile range (IQR) 88.94-113.26) than those in the control group (median 86.40; IQR 75.26-96.92; $p<0.001$ ) (Fig. 1). After adjusting this difference for gestational age at blood sampling and BMI, afamin concentrations remained higher in 


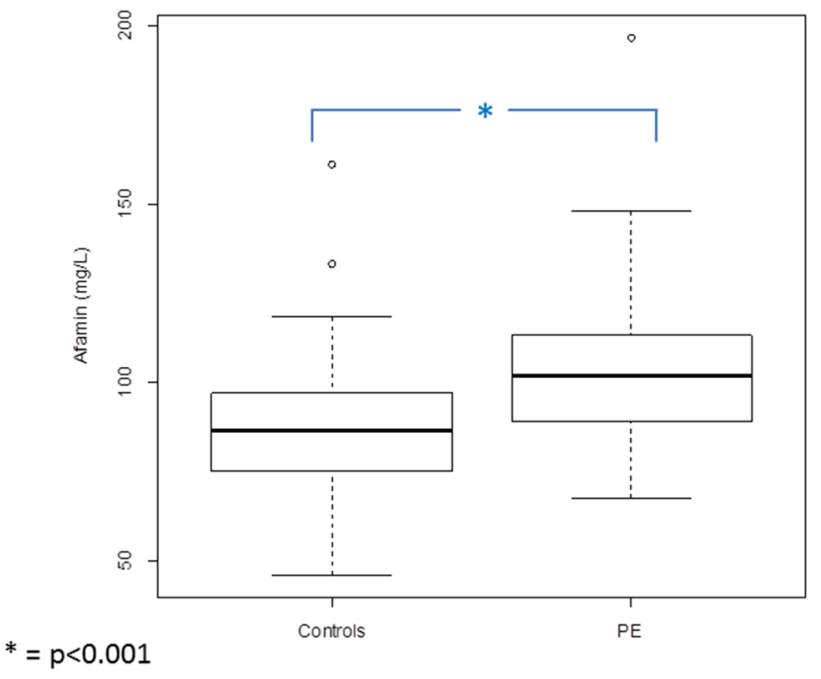

Fig. 1 Boxplots illustrating the distribution of afamin concentrations in patients in the first trimester of pregnancy developing PE during pregnancy $(n=39)$ compared to control subjects without pregnancy complications $(n=98)$

patients developing PE than in the control group [odds ratio per afamin standard deviation: 1.60 (95\% CI: $1.04-2.58 ; p=0.04)]$.

Receiver operating characteristic (ROC) analysis showed that a serum afamin concentration of $87.8 \mathrm{mg} / \mathrm{L}$ had the best sensitivity (79.5\%) and specificity (57.1\%) to discriminate between women in whom PE will develop during their pregnancy and those with uncomplicated pregnancy. The area under the curve (AUC) was 0.73 (95\% CI: 0.63-0.82) (Fig. 2).

\section{Subgroup analysis of patients who developed early-onset and late-onset PE}

Patient characteristics of both subgroups are shown in Table 1. Subgroups differed significantly between gestational age at delivery and newborn weight and with borderline significance between age and PAPP-A.

Afamin concentrations were highest in women developing late-onset PE (median 105.10; IQR 89.78-116.17), followed by levels of women developing early-onset PE (median 93.68; IQR 88.03-102.00) and lowest in women without PE (median 86.40; IQR 75.26-96.92) (Fig. 3). Afamin values differed statistically differently between women who developed late-onset PE and controls $(p<0.001)$. The difference between afamin concentrations in women who developed late-onset and early-onset $\mathrm{PE}$ and between early-onset PE and controls was not statistically different ( $p=0.20$ and $p=0.10$, respectively).

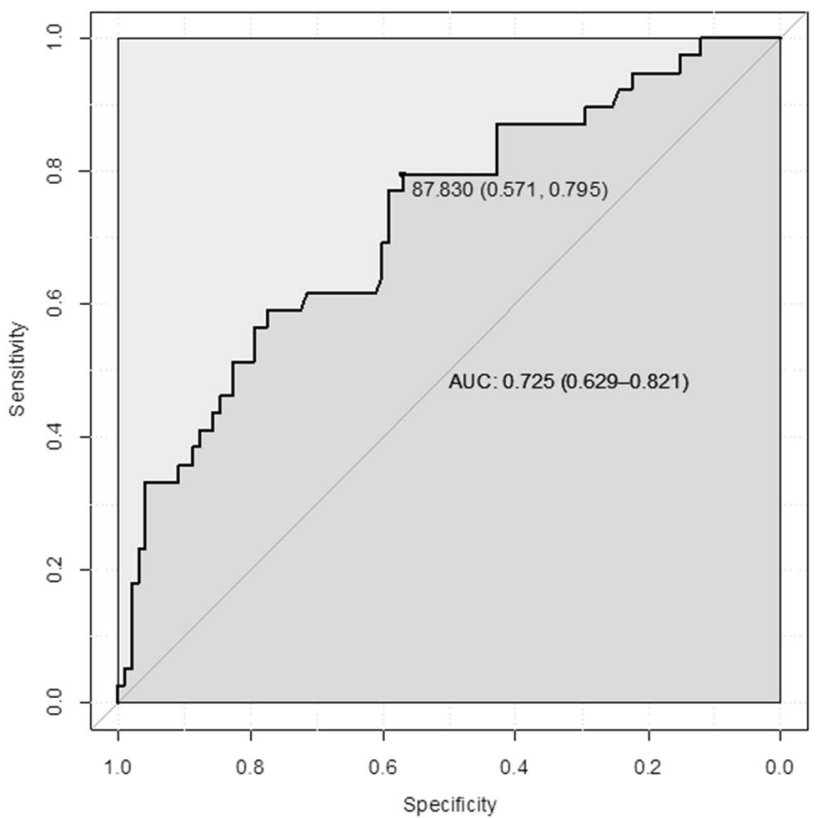

Fig. 2 Receiver operating characteristics (ROC) analysis demonstrating specificity and sensitivity of afamin concentrations measured during the first trimester to discriminate between patients developing PE and women without pregnancy complications

\section{Late-onset PE}

After adjusting these differences for gestational age at blood sampling and BMI, afamin concentrations remained higher in patients developing late-onset PE than in the control

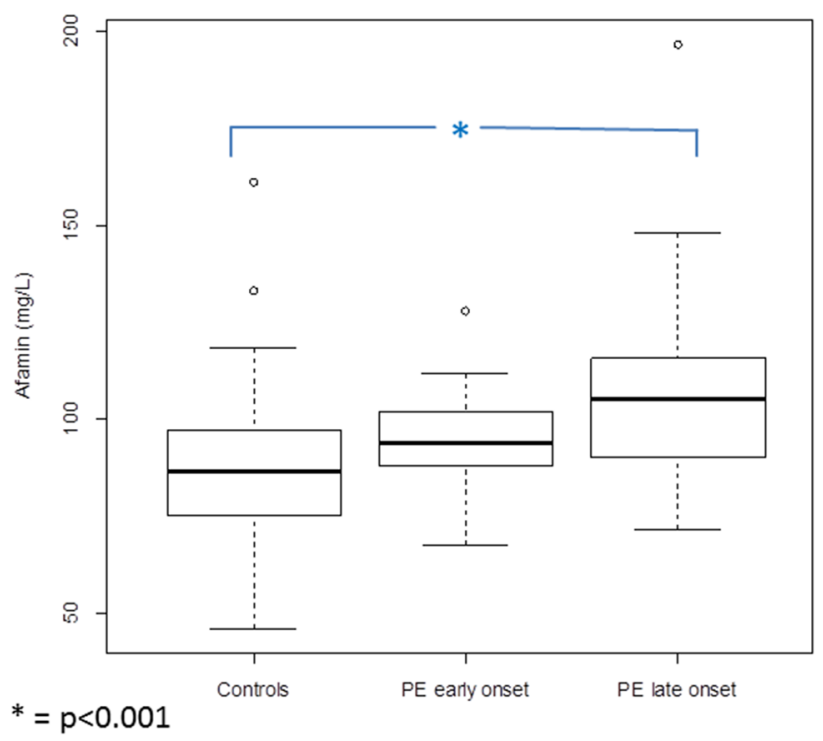

Fig. 3 Boxplots illustrating the distribution of afamin concentrations in patients in the first trimester of pregnancy developing early-onset PE $(n=10)$ and late-onset PE $(n=29)$ during pregnancy compared to control subjects without pregnancy complications $(n=98)$ 
group [odds ratio per afamin standard deviation: 1.62 (95\% CI: $0.98-2.70 ; p=0.06)]$. Although statistical significance was not reached, the odds ratio remained identical compared to the analysis of the whole cohort (early- and late-onset PE).

ROC analysis showed that a serum afamin concentration of $97.2 \mathrm{mg} / \mathrm{L}$ had the best sensitivity (65.5\%) and specificity (77.6\%) to discriminate between women in whom late-onset PE will develop during pregnancy and those with uncomplicated pregnancy. The area under the curve (AUC) was 0.75 (95\% CI: 0.64-0.86) (Fig. 4).

\section{Early-onset PE}

After adjusting the results for gestational age and BMI, afamin concentrations remained only slightly higher in patients with developing early-onset PE than in the control group [odds ratio per afamin standard deviation 1.18 (95\% CI: $0.61-2.27 ; p=0.62)$ ].

ROC analysis showed that a serum afamin concentration of $87.8 \mathrm{mg} / \mathrm{L}$ had the best sensitivity $(80.0 \%)$ and specificity $(57.1 \%)$ to discriminate between women in whom earlyonset PE will develop during their pregnancy and those with uncomplicated pregnancy. The area under the curve (AUC) was 0.66 (95\% CI: 0.49-0.83) (Fig. 5).

Choosing a cut-off value of $106.9 \mathrm{mg} / \mathrm{L}$ and accepting a false-positive rate of $10 \%$, the detection rate of all PE cases

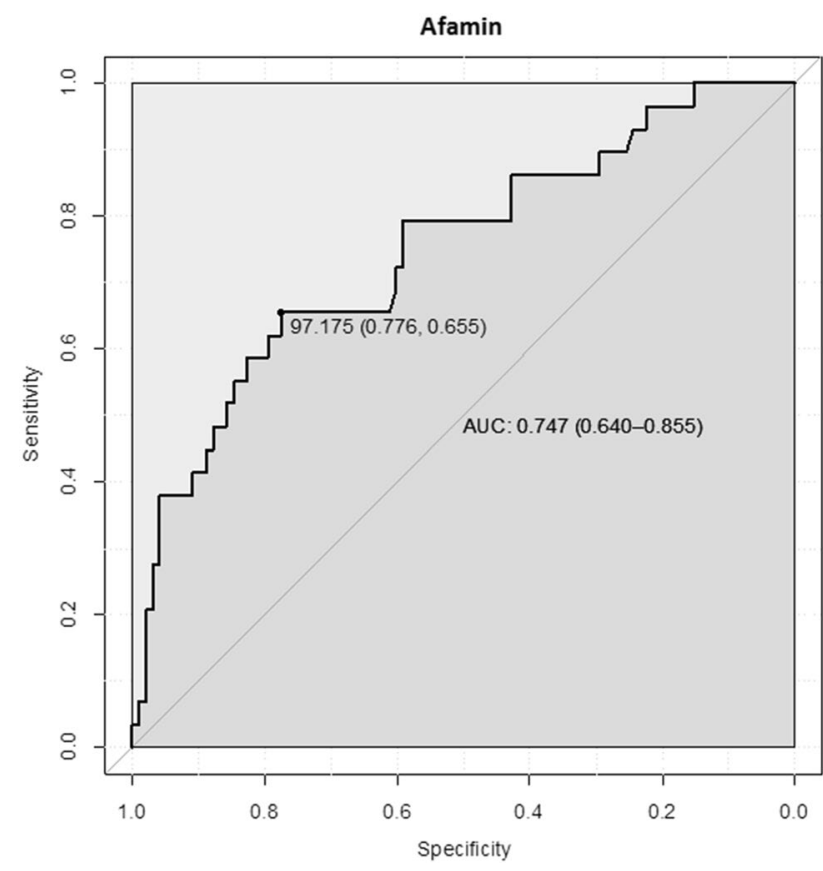

Fig. 4 Receiver operating characteristics (ROC) analysis demonstrating specificity and sensitivity of afamin concentrations measured during the first trimester to discriminate between patients developing late-onset PE and women without pregnancy complications

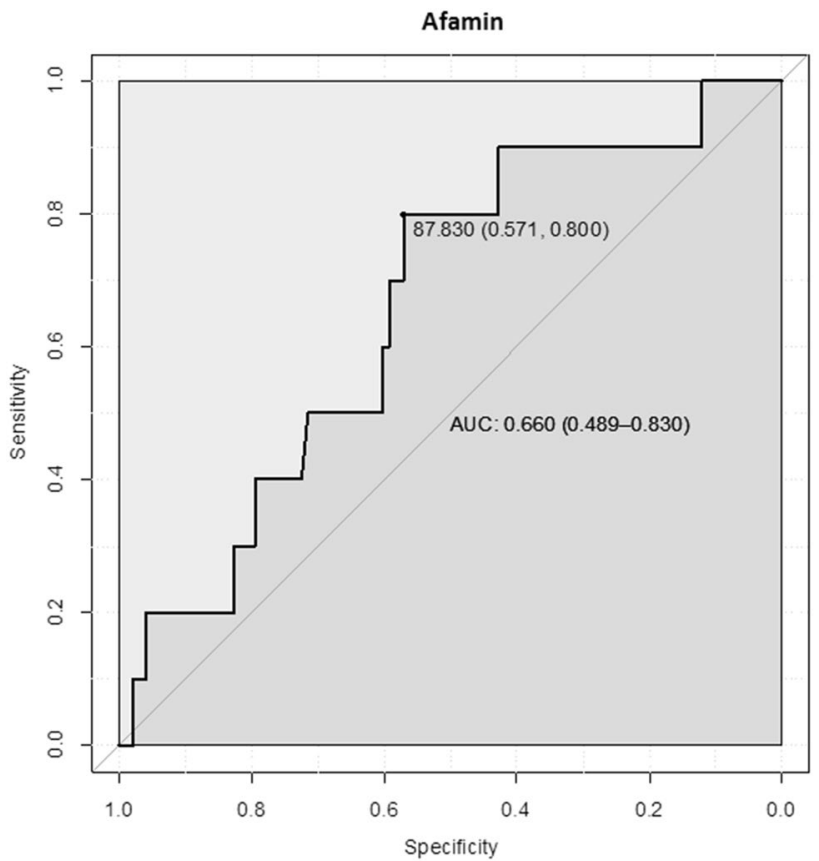

Fig. 5 Receiver operating characteristics (ROC) analysis demonstrating specificity and sensitivity of afamin concentrations measured during the first trimester to discriminate between patients developing early-onset PE and women without pregnancy complications

was $35.9 \%$; of late-onset PE, it was $41.4 \%$ and of early-onset PE 20\%, respectively.

\section{Discussion}

In the present study, we compared serum afamin concentrations measured in the first trimester in 39 pregnant women developing PE in the course of their pregnancy with those of 98 women with uncomplicated pregnancies. Afamin concentrations were significantly higher in patients developing PE than in healthy control subjects. An afamin threshold concentration of $87.8 \mathrm{mg} / \mathrm{L}$ exhibited a sensitivity and specificity of 79.5 and $57.1 \%$, respectively, discriminating between those two groups. In the study cohort, a high percentage of women (75\%) developed late-onset PE. Subgroup analysis revealed that patients developing late-onset PE, but not those developing early-onset PE, showed substantially higher afamin concentrations compared to controls in the first trimester.

Our work confirms and extends a recently published study [22] also reporting significantly increased afamin concentrations in the first trimester in patients with developing PE compared to control subjects. The strength of our analysis is the subgroup analysis regarding late- and early-onset PE and the higher number of preeclamptic patients. 
In accordance with the previous studies of our working group [19, 21], we found generally higher afamin concentrations in pregnant as well as in non-pregnant women compared to other study cohorts. This phenomenon remains somehow inexplicable and we hypothesise that ethnic differences might contribute to the higher concentrations in our study population.

Afamin is thought to reflect a condition of oxidative stress, since elevated concentrations correlate with features of the metabolic syndrome, especially IR and T2DM [17, 19]. Afamin concentrations were also reported to be positively correlated with IR in PCOS patients [19]. Oxidative stress, however, seems to be a concomitant factor in metabolic syndrome, IR and PCOS [31-33]. It was already demonstrated that preeclampsia is a status of elevated oxidative stress [10, 34]. Additionally, patients with placental dysfunction are at high risk to develop metabolic syndrome, IR [8] and cardiovascular disease later in their life [35].

$75 \%$ of our preeclamptic patients, examined in the first trimester, developed late-onset PE. It was hypothesised that the pathogenesis of late-onset PE differs from the early-onset form. Early-onset PE seems to be caused by disturbed trophoblast invasion and the late-onset form rather results from adverse maternal conditions [7, 36]. Redman et al. hypothesised that late-onset PE derives from a limited intervillous perfusion resulting in intravillous hypoxia, oxidative stress and cell damage [6]. In conclusion, elevated afamin values may indicate a status of maternal oxidative stress which could mainly limit the placental function in the last trimester of pregnancy rather than disturbed early trophoblast invasion. Since afamin is not secreted by the placenta [27], elevated afamin concentrations more likely reflect a maternal contribution to the pathophysiology, e.g. a limited maternal vascular capacity [7].

The different pathophysiology is also reflected by the potency to predict and prevent placental disorders during the first trimester. Only early-onset PE can be predicted during the first trimester [4] and prevented by Aspirin ${ }^{\circledR}$ intake at a $>90 \%$ percentage $[2,3]$. In contrast, the number of $\mathrm{PE}$ cases of all trimesters which require delivery before the 42nd gestational week is predictable only in $54 \%$ of cases using biochemical and clinical parameters [37]. In our study, the detection rate of all PE cases (early- and late onset PE) was $36 \%$ using a cut-off value of $106.9 \mathrm{mg} / \mathrm{L}$, with a false-positive rate of $10 \%$. Regarding the late-onset PE cases only, the detection rate was $41.4 \%$, respectively, using the same cut-off value and accepting a false-positive rate of $10 \%$. This result is comparable to the predictive power of PIGF as a single parameter determined during the first trimester, which allows to detect $40 \%$ of PE cases by accepting a false-positive rate of $10 \%$ [37]. Further research with higher study numbers is recommended to build an algorithm which refers to a substantially higher detection rate of afamin concentrations by additional inclusion of the patient's history and clinical features.

Because our early-onset PE subgroup was very small, our results cannot finally exclude an important role of oxidative stress in the pathogenesis of early-onset PE. Furthermore, clinical experience elucidates that the early- and the late-onset PE cannot be separated strictly and overlapping pathophysiological mechanisms are most likely. Physiological oxidative stress is an important component of normal foetal and placental development [38]. With placental maturation at the end of the first trimester, the oxygen level increases and reactive oxygen species (ROS) are produced. ROS themselves are responsible for an adequate trophoblast regression to cause the discoid placenta. Overproduction of ROS is associated with accelerated trophoblast regression resulting in placental dysfunction $[39,40]$. In conclusion, the increased afamin concentrations found in first-trimester patients may reflect the present oxidative stress, which also could influence the placental differentiation at this step.

\section{Conclusion}

As a consequence of our study, afamin concentrations determined in the first trimester may identify women at high risk of pregnancy complications associated with maternal oxidative stress and chronic inflammation such as PE. Elevated afamin values are hypothesised to reflect a maternal rather than a placental condition. Therefore, it is not surprising that elevated afamin concentrations identify women developing late-onset PE. Since afamin concentrations are independent of age, fasting state and daily fluctuations [29], the determination appears to indicate a very suitable and inexpensive new biomarker.

Since afamin concentrations mainly reflect a maternal condition, further research is recommended to explore the association between afamin determined before conception and the occurrence of pregnancy complications. Such a preconception risk profiling of women who want to get pregnant may offer an effective prevention of pregnancy complications by life-style modification or tailored early pregnancy care.

Acknowledgements Open access funding provided by University of Innsbruck and Medical University of Innsbruck. We thank the laboratory staff members Bernd Linke, Sieglinde Arndt, Ute Kirsch, Claudine Kühn, Jens Rasch, Gabriele Sehn and our nurses for excellent technical assistance and data documentation. We are grateful to Dr. Florence Witte for English editing and critical reading of the manuscript. This work was funded by the University of Duisburg-Essen to AK and the Austrian Research Fund (P19969-B11) to HD.

Author contributions AK: Project development, data collection, data analysis and management, manuscript writing; AE: data collection, manuscript writing; PM: data collection, DA: data management, BS: 
data analysis, manuscript editing, MF: data analysis, CB: protocol development, manuscript writing, RK: project development, manuscript editing, AG: biochemical analysis, manuscript editing, HD: Project development, data management, manuscript editing.

\section{Compliance with ethical standards}

Conflict of interest All authors declare no conflicts of interest.

Ethical approval This study was approved by the research ethics committee of the University of Duisburg-Essen (number 125212-BO) and performed in accordance with the ethical standards as laid down in the 1964 Declaration of Helsinki and its later amendments.

Informed consent All women provided written informed consent.

Open Access This article is distributed under the terms of the Creative Commons Attribution 4.0 International License (http://creativeco mmons.org/licenses/by/4.0/), which permits unrestricted use, distribution, and reproduction in any medium, provided you give appropriate credit to the original author(s) and the source, provide a link to the Creative Commons license, and indicate if changes were made.

\section{References}

1. Purde MT, Baumann M, Wiedemann U, Nydegger UE, Risch L, Surbek D, Risch M (2015) Incidence of preeclampsia in pregnant Swiss women. Swiss Med Wkly 145:w14175

2. Roberge S, Villa P, Nicolaides K, Giguere Y, Vainio M, Bakthi A, Ebrashy A, Bujold E (2012) Early administration of low-dose aspirin for the prevention of preterm and term preeclampsia: a systematic review and meta-analysis. Fetal Diagn Ther 31:141-146

3. Poon LC, Wright D, Rolnik DL, Syngelaki A, Delgado JL, Tsokaki T, Leipold G, Akolekar R, Shearing S, De Stefani L, Jani JC, Plasencia W, Evangelinakis N, Gonzalez-Vanegas O, Persico N, Nicolaides KH (2017) Aspirin for evidence-based preeclampsia prevention trial: effect of aspirin in prevention of preterm preeclampsia in subgroups of women according to their characteristics and medical and obstetrical history. Am J Obstet Gynecol 217:585

4. Akolekar R, Syngelaki A, Poon L, Wright D, Nicolaides KH (2013) Competing risks model in early screening for preeclampsia by biophysical and biochemical markers. Fetal Diagn Ther 33:8-15

5. O'Gorman N, Wright D, Syngelaki A, Akolekar R, Wright A, Poon LC, Nicolaides KH (2016) Competing risks model in screening for preeclampsia by maternal factors and biomarkers at 11-13 weeks gestation. Am J Obstet Gynecol 214:103

6. Redman CW, Sargent IL, Staff AC (2014) IFPA Senior Award Lecture: making sense of pre-eclampsia-two placental causes of preeclampsia? Placenta 35(Suppl):S20-S25

7. Valensise H, Vasapollo B, Gagliardi G, Novelli GP (2008) Early and late preeclampsia: two different maternal hemodynamic states in the latent phase of the disease. Hypertension 52:873-880

8. Hooijschuur MC, Ghossein-Doha C, Al-Nasiry S, Spaanderman ME (2015) Maternal metabolic syndrome, preeclampsia, and small for gestational age infancy. Am J Obstet Gynecol 213(370):e371-e377

9. Ness RB, Sibai BM (2006) Shared and disparate components of the pathophysiologies of fetal growth restriction and preeclampsia. Am J Obstet Gynecol 195:40-49
10. Oztas E, Ozler S, Tokmak A, Erel O, Ergin M, Uygur D, Danisman N (2016) Oxidative stress markers in severe preeclampsia and preeclampsia-related perinatal morbidity—preliminary report. Ginekol Pol 87:436-441

11. Yinon Y, Kingdom JC, Odutayo A, Moineddin R, Drewlo S, Lai V, Cherney DZ, Hladunewich MA (2010) Vascular dysfunction in women with a history of preeclampsia and intrauterine growth restriction: insights into future vascular risk. Circulation 122:1846-1853

12. Voegele AF, Jerkovic L, Wellenzohn B, Eller P, Kronenberg F, Liedl KR, Dieplinger H (2002) Characterization of the vitamin E-binding properties of human plasma afamin. Biochemistry 41:14532-14538

13. Lichenstein HS, Lyons DE, Wurfel MM, Johnson DA, McGinley MD, Leidli JC, Trollinger DB, Mayer JP, Wright SD, Zukowski MM (1994) Afamin is a new member of the albumin, alpha-fetoprotein, and vitamin D-binding protein gene family. J Biol Chem 269:18149-18154

14. Niki E, Traber MG (2012) A history of vitamin E. Ann Nutr Metab 61:207-212

15. Jerkovic L, Voegele AF, Chwatal S, Kronenberg F, Radcliffe CM, Wormald MR, Lobentanz EM, Ezeh B, Eller P, Dejori N, Dieplinger B, Lottspeich F, Sattler W, Uhr M, Mechtler K, Dwek RA, Rudd PM, Baier G, Dieplinger H (2005) Afamin is a novel human vitamin E-binding glycoprotein characterization and in vitro expression. J Proteome Res 4:889-899

16. Seeber BE, Czech T, Buchner H, Barnhart KT, Seger C, Daxenbichler G, Wildt L, Dieplinger H (2010) The vitamin E-binding protein afamin is altered significantly in the peritoneal fluid of women with endometriosis. Fertil Steril 94:2923-2926

17. Kronenberg F, Kollerits B, Kiechl S, Lamina C, Kedenko L, Meisinger C, Willeit J, Huth C, Wietzorrek G, Altmann ME, Thorand B, Melmer A, Dahnhardt D, Santer P, Rathmann W, Paulweber B, Koenig W, Peters A, Adham IM, Dieplinger H (2014) Plasma concentrations of afamin are associated with the prevalence and development of metabolic syndrome. Circ Cardiovasc Genet 7:822-829

18. Kollerits B, Lamina C, Huth C, Marques-Vidal P, Kiechl S, Seppala I, Cooper J, Hunt SC, Meisinger C, Herder C, Kedenko L, Willeit J, Thorand B, Dahnhardt D, Stockl D, Willeit K, Roden M, Rathmann W, Paulweber B, Peters A, Kahonen M, Lehtimaki T, Raitakari OT, Humphries SE, Vollenweider P, Dieplinger H, Kronenberg F (2017) Plasma concentrations of afamin are associated with prevalent and incident type 2 diabetes: a pooled analysis in more than 20,000 individuals. Diabetes Care 40:1386-1393

19. Koninger A, Edimiris P, Koch L, Enekwe A, Lamina C, KasimirBauer S, Kimmig R, Dieplinger H (2014) Serum concentrations of afamin are elevated in patients with polycystic ovary syndrome. Endocr Connect 3:120-126

20. Seeber B, Morandell E, Lunger F, Wildt L, Dieplinger H (2014) Afamin serum concentrations are associated with insulin resistance and metabolic syndrome in polycystic ovary syndrome. Reprod Biol Endocrinol 12:88

21. Koninger A, Mathan A, Mach P, Frank M, Schmidt B, Schleussner E, Kimmig R, Gellhaus A, Dieplinger H (2018) Is afamin a novel biomarker for gestational diabetes mellitus? A pilot study. Reprod Biol Endocrinol 16:30

22. Tramontana A, Dieplinger B, Stangl G, Hafner E, Dieplinger H (2018) First trimester serum afamin concentrations are associated with the development of pre-eclampsia and gestational diabetes mellitus in pregnant women. Clin Chim Acta 476:160-166

23. Founds SA, Catov JM, Gallaher MJ, Harger GF, Markovic N, Roberts JM (2011) Is there evidence of separate inflammatory or metabolic forms of preeclampsia? Hypertens Pregnancy 30:1-10

24. Ozcan U, Cao Q, Yilmaz E, Lee AH, Iwakoshi NN, Ozdelen E, Tuncman G, Gorgun C, Glimcher LH, Hotamisligil GS (2004) 
Endoplasmic reticulum stress links obesity, insulin action, and type 2 diabetes. Science 306(5695):457-461

25. Scioscia M, Karumanchi SA, Goldman-Wohl D, Robillard PY (2015) Endothelial dysfunction and metabolic syndrome in preeclampsia: an alternative viewpoint. J Reprod Immunol 108:42-47

26. Thadhani R, Ecker JL, Mutter WP, Wolf M, Smirnakis KV, Sukhatme VP, Levine RJ, Karumanchi SA (2004) Insulin resistance and alterations in angiogenesis: additive insults that may lead to preeclampsia. Hypertension 43:988-992

27. Hubalek M, Buchner H, Mortl MG, Schlembach D, Huppertz B, Firulovic B, Kohler W, Hafner E, Dieplinger B, Wildt L, Dieplinger $\mathrm{H}$ (2014) The vitamin E-binding protein afamin increases in maternal serum during pregnancy. Clin Chim Acta 434:41-47

28. Practice ACOG (2002) ACOG practice bulletin. Diagnosis and management of preeclampsia and eclampsia. Number 33, January 2002. American College of Obstetricians and Gynecologists. Int J Gynecol Obstet 77:67-75

29. Dieplinger B, Egger M, Gabriel C, Poelz W, Morandell E, Seeber B, Kronenberg F, Haltmayer M, Mueller T, Dieplinger H (2013) Analytical characterization and clinical evaluation of an enzymelinked immunosorbent assay for measurement of afamin in human plasma. Clin Chim Acta 425:236-241

30. R Core Team. The R project for statistical computing. R Foundation for Statistical Computing, Vienna. http://www.R-project.org/. Accessed 30 Nov 2016

31. Desai V, Prasad NR, Manohar SM, Sachan A, Narasimha SR, Bitla AR (2014) Oxidative stress in non-obese women with polycystic ovarian syndrome. J Clin Diagn Res 8:CC01-03

32. Ferroni P, Basili S, Falco A, Davi G (2004) Inflammation, insulin resistance, and obesity. Curr Atheroscler Rep 6:424-431
33. Gonzalez F, Rote NS, Minium J, Kirwan JP (2006) Reactive oxygen species-induced oxidative stress in the development of insulin resistance and hyperandrogenism in polycystic ovary syndrome. J Clin Endocrinol Metab 91:336-340

34. Turpin CA, Sakyi SA, Owiredu WK, Ephraim RK, Anto EO (2015) Association between adverse pregnancy outcome and imbalance in angiogenic regulators and oxidative stress biomarkers in gestational hypertension and preeclampsia. BMC Pregnancy Childbirth 15:189

35. Bellamy L, Casas JP, Hingorani AD, Williams DJ (2007) Preeclampsia and risk of cardiovascular disease and cancer in later life: systematic review and meta-analysis. BMJ 335(7627):974

36. Redman CW, Sargent IL (2009) Placental stress and pre-eclampsia: a revised view. Placenta 30(Suppl A):S38-S42

37. Poon LC, Nicolaides KH (2014) Early prediction of preeclampsia. Obstet Gynecol Int 2014:297397

38. Torres-Cuevas I, Parra-Llorca A, Sanchez-Illana A, NunezRamiro A, Kuligowski J, Chafer-Pericas C, Cernada M, Escobar J, Vento M (2017) Oxygen and oxidative stress in the perinatal period. Redox Biol 12:674-681

39. Burton GJ, Jauniaux E (2011) Oxidative stress. Best Pract Res Clin Obstetrics Gynaecol 25:287-299

40. Jauniaux E, Hempstock J, Greenwold N, Burton GJ (2003) Trophoblastic oxidative stress in relation to temporal and regional differences in maternal placental blood flow in normal and abnormal early pregnancies. Am J Pathol 162:115-125

41. Voigt M, Fusch C, Olbertz D, Hartmann K, Rochow N, Renken C, Schneider KTM (2006) Analysis of detailed percentiles for the body measurement of newborns. Geburtsh Frauenheilk 66:956-970 